\section{Fortbildung im Fokus}

er Deutsche Allergiekongress 2010 der drei deutschen allergologischen Fachgesellschaften trägt erstmals nicht mehr den Titel "Gemeinsam" - zu selbstverständlich ist die Zusammenarbeit von ÄDA, DGAKI und GPA inzwischen geworden, als dass sie noch extra erwähnt werden müsste. Veranstaltungsort 2010 wird das Convention Center der Messe Hannover sein, das Kongressthema lautet „Allergologie im Wandel“".

Zielgruppe des Kongresses sind niedergelassene Ärzte, Klinikärzte und Forscher. Das bisherige Ziel der Allergiekongresse, Informationen zu aktuellen klinischen und wissenschaftlichen Entwicklungen zu präsentieren, soll beibehalten werden. Aufgrund der Rückmeldungen zu den Veranstaltungen der letzten Jahre soll 2010 jedoch noch stärker als bisher - insbesondere an den Kongresstagen Freitag und Samstag der Fortbildungsaspekt für klinisch täti-

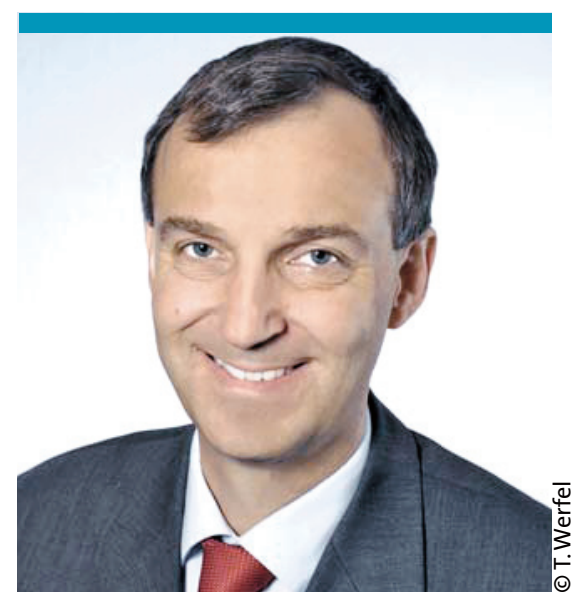

Prof. Dr. Thomas Werfel, Klinik für Dermatologie und Venerologie, Medizinische Hochschule Hannover

ge Ärzte in Praxen und Krankenhäusern betont werden.

Unterschiedliche und zum Teil neue Veranstaltungsformen sollen den geänderten Ansprüchen gerecht werden und Sie motivieren, den Kongress in Hannover zu besuchen. Auch möchte ich Sie herzlich dazu einladen, selbst Beiträge für die Posterdiskussionen, die wissenschaftlichen oder auch die kasuistischen Sitzungen einzureichen - spätestens bis 31. Mai 2010. Ein abwechslungsreiches Abendprogramm mit einer feierlichen Kongresseröffnung auf dem Messegelände und anschließendem Beisammensein am Mittwochabend, einer zwanglosen Allergieparty am Donnerstag und einem Festabend am Freitag im Alten Rathaus soll Ihnen einen anregenden Ausklang der Kongresstage und einen kollegialen Austausch in schöner Atmosphäre ermöglichen.

Ich freue mich, Sie im September 2010 in Hannover begrüßen zu dürfen!

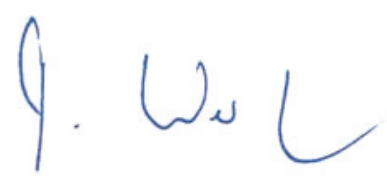

Prof. Dr. Thomas Werfel

Kongresspräsident

\section{Highlights in Hannover}

\section{Fortbildungssymposien}

Donnerstag, 9. September 2010

_ Antiallergische Pharmakotherapie

—Die persistierende Rhinitis: allergisch oder nicht?

— Langfristiges Management bei Patienten mit Anaphylaxie

Freitag, 10. September 2010

$-1 \times 1$ der IgE-Diagnostik

— Atopische Dermatitis

— Nahrungsmittelallergie

- Chronische Urtikaria und Angioödeme

_ Spezifische Immuntherapie mit Inhalationsallergenen

— Allergisches Asthma bronchiale

Samstag, 11. September 2010

_ Allergische Rhinitis und Konjunktivitis

—Allergisches Kontaktekzem

_ Insektengiftallergie

- Praktisches Vorgehen bei Arzneimittelallergien

— Besonderheiten bei Allergien im Kindesalter

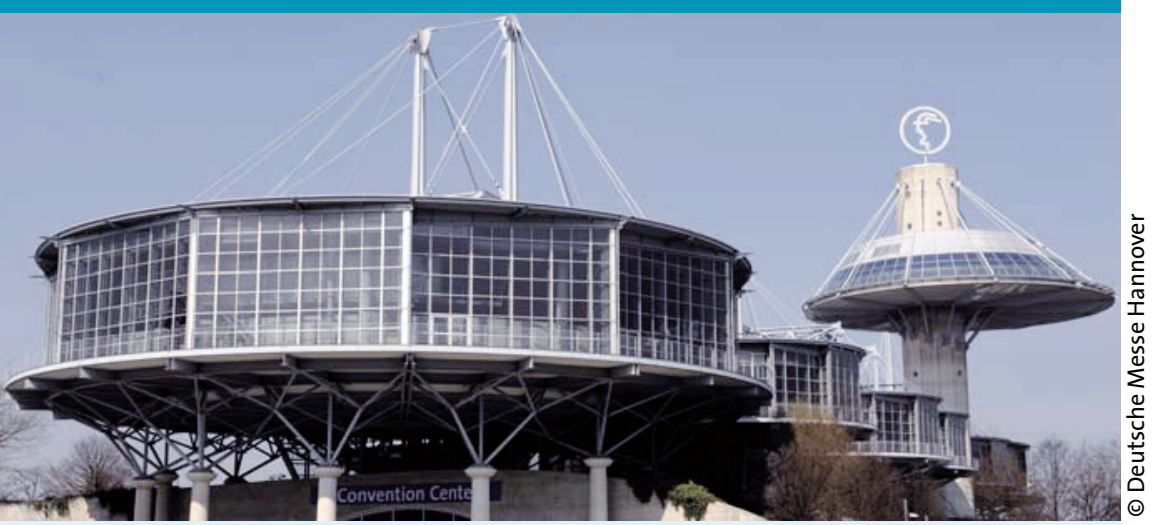

\section{Plenarveranstaltungen}

- Allergologie im Wandel

- Therapie allergischer Erkrankungen in unterschiedlichen Lebensphasen

— Genetik allergischer Erkrankungen

_ Neue Allergene

- Neuroimmunologie in der Allergie

- Das Neueste zur Prävention und Therapie allergischer Erkrankungen

Weitere Veranstaltungsformen

— Klinisch-wissenschaftliche Symposien

_ Interaktive Seminare
— Pro-und-Kontra-Sitzungen

_ Grundlagenorientierte wissenschaftliche Symposien

— Die fünf interessantesten klinischen Publikationen des letzten Jahres

_ Industriesymposien

_ Sherlock Holmes in der Allergologie (Auswahl der besten Kasuistiken aus den eingegangenen Originalbeiträgen)

- Originalbeiträge aus Klinik und Forschung

— Geführte Posterdiskussionen

_ Praktische Kurse und Seminare 